\title{
La Formación Investigativa Como Salida Para El Compromiso Profesional De Futuros Profesores En Angola
}

\section{Eugenio Figueiredo}

Profesor colaborador y asociado en la Escuela Superior Pedagógica de Bié, Angola

\section{Dña. Miriam Agreda Montoro}

Diplomada en Magisterio de Educación Especial, Licenciada en Pedagogía, Doctora en Ciencias de la Educación y Máster Oficial de Formación del Profesorado de Secundaria y Bachillerato, Formación Profesional y Enseñanza de Idiomas, especialidad Orientación Educativa. Profesora colaboradora en la Universidad Internacional de la Rioja, Facultad de Educación (España).

\section{Dña. María Angustias Hinojo Lucena}

Diplomada en Magisterio de Educación Primaria, Licenciada en Pedagogía. Docente e investigadora del departamento de Didáctica y Organización Escolar de la Universidad de Granada. Facultad de Ciencias de la Educación, Granada (España).

\begin{abstract}
This paper aims to enhance the research carried out on teacher training as a current need in Angola's Higher Education due to the lack of any practical introduction to research as part of an essential mode of action in professional training. This paper uses references provided in social sciences as a basis to inform about the importance of research training during the educational process of both students and teachers in Angola.. We conclude there is a need to propose policies that create profound changes in the training model of education practitioners so as to include an efficient general knowledge as well as the ability to develop proper research.
\end{abstract}

Keywords: research training, teaching skill, initial training, long-life learning, professional identity

\section{Resumen}

El presente artículo pretende realzar la formación investigativa de los profesores como una necesidad actual en la enseñanza superior de Angola, 
por la carencia práctica de introducción de la investigación como parte de esencial del modo de actuación del profesional de la educación. Su objetivo es fundamentar, desde los referentes que brindan las Ciencias Sociales, la importancia de la formación investigativa en el proceso de formación inicial y continua de los estudiantes y profesores de las escuelas pedagógicas de Angola. Las conclusiones reflejan la necesita plantear políticas que generen cambios profundos en el modelo de formación de profesionales de la educación que incluya una eficiente educación general y capacidad para investigar.

Palabras clave: formación investigativa, competencia docente, formación inicial, aprendizaje a lo largo de la vida, identidad profesional

\section{Introducción}

El papel relevante dado a la investigación como proceso sustantivo y vinculado a la práctica educativa y, por tanto, a la calidad de la educación mediante la construcción de conocimientos ha supuesto muchos cambios en la Educación Superior contemporánea, ya que tiene la obligación de responder a las necesidades específicas que surgen en la sociedad. También se vincula de manera extensiva en cuanto a la consecución de logros y objetivos para dar solución a los problemas contextuales o con un mayor alcance, requiriendo los recursos de los que la ciencia se provee.

Angola no es un país ajeno a esta situación, por lo que para mejorar todo el contexto de la Educación Superior del país, se está trabajando en profundas reformas a través del planteamiento de políticas que generen cambios en el modelo de formación de profesores, sobre todo en las escuelas pedagógicas angolanas. Este nuevo modelo formativo debe incluir una educación eficaz y eficiente, teniendo como base las habilidades investigativas, aprender a aprender, reciclaje y actualización pedagógica para un mundo en constante cambio. Así mismo, el profesorado deberá dinamizar el proceso de enseñanza aprendizaje, proporcionar los recursos necesarios para la adquisición de competencias del alumnado, haciendo especial hincapié en aquellas que posibilitan la construcción y gestión de su propio aprendizaje y conocimiento.

A este respecto encontramos deficiencias y carencias en la realidad educativa angolana, generándose obstáculos para el logro de las metas planteadas. Por consiguiente, la introducción de la investigación como parte esencial de la práctica profesional del profesorado es esencial para encaminar y apoyar el proceso de creación y construcción de nuevos conocimientos científicos y pedagógicos, con el fin de atender a la demanda social naciente en el contexto angolano. 
En sentido general, el problema de la preparación de los docentes en la enseñanza superior es una de las mayores preocupaciones del órgano de tutela del país, el Ministerio de la Enseñanza Superior, que propone la formación continua del profesional y la adquisición del título académico de master o el grado científico de doctor a partir de un régimen de trabajo a tiempo total o parcial que redunde positivamente en su desempeño profesional.

En la actualidad, los profesores másteres y doctores constituyen una cantidad insignificante dentro de las instituciones de enseñanza superior (IES) que existen; los pocos que hay, en su mayoría, están concentrados en la capital de Angola, Luanda. Generalmente, en referencia al régimen de trabajo en general, la mayor parte de los docentes imparte clases en varias IES o está vinculado a otro organismo. Son pocos los que ejercen la profesión docente, por ello, es muy difícil lograr un buen desempeño en esas circunstancias de trabajo. Esa situación tributa de forma comprometedora a la calidad del proceso.

En las escuelas superiores pedagógicas la situación no es muy diferente, con la particularidad de tener pocos profesores nacionales a tiempo total y con poca experiencia en la docencia de la enseñanza superior. Esa es una gran preocupación del gobierno intenta implementar el Plan de Formación de Cuadros, cuya prioridad es la formación de profesores de la enseñanza superior en estudios de maestrías y doctorados.

A pesar de la implantación de este plan, en estos momentos se plantea un problema sobre el presente y es qué hacer ahora, ¿esperar a que el país posea el número suficiente de másteres y doctores? ¿buscar soluciones a partir de la superación de los estudios de posgrado?. Si la solución es la de enmendar justo después de la superación de los estudios, se origina otra cuestión, ¿cómo hacerlo?.

Para eso, se considera necesario tener en cuenta la investigación pedagógica como componente esencial que modela el modo de actuación del profesional, teniendo en cuenta las políticas, la cultura y la competencia investigativa que se requiere en una institución de formación de profesores de excelencia. De ahí entonces que el presente trabajo aborde como problema social la necesidad de la formación investigativa en el proceso de formación inicial y continua de estudiantes y profesores en las escuelas pedagógicas de Angola. Se plantea como objetivo principal fundamentar la importancia de la formación investigativa de profesores y estudiantes en las escuelas pedagógicas de Angola. 


\section{La formación de los docentes en investigación para el desarrollo de una actitud de compromiso profesional.}

Uno de las mayores retos y desafíos en la Educación Superior ha sido otorgar a la investigación su papel dentro de la formación base del profesional de la educación. Castellanos (1998:23 asume que “a nivel mundial se están examinando en las últimas décadas múltiples alternativas para no elitizar la investigación educativa y superar la desvinculación existente entre esta y el trabajo educativo en la escuela”. Se trata de abrir los espacios a formas más democráticas de la construcción del conocimiento desde la cooperación y colaboración para utilizarlo en la transformación de la realidad educativa.

En este mismo sentido, entre varios aspectos debatidos en la Conferencia Mundial sobre la Educación Superior realizada en París en octubre de 1998, al declarar sus funciones y misiones, se tuvo en cuenta el papel de la investigación.

Artículo 1. La misión de educar, formar y realizar investigaciones.

(...) Construir un espacio abierto para la educación superior que propicie el aprendizaje permanente (...) formar ciudadanos que participen activamente en la sociedad. Promover y difundir el conocimiento por medio de la enseñanza, la actividad académica y la investigación (...) generando conocimientos gracias a la investigación, fomentando y desarrollando la investigación científica y tecnológica a la par que la investigación especializada en el campo de las ciencias sociales y las humanidades ( ...) (UNESCO 1998:4).

En estos pensamientos se refleja la necesidad de una enseñanza superior proactiva, capaz de generar profesionales que atiendan a las exigencias sociales actuales y que les permita el poder proyectar el futuro desde su presente.

En Angola, se demanda formas a los profesionales en investigación desde un enfoque de educación permanente con base esencial en el proceso de enseñanza-aprendizaje y extrapolado a aspectos extracurriculares trabajando en proyectos concretos. Esto implica una preparación en investigación de los profesores, asumiéndola como proceso integrador entre el componente académico y el laboral y como vía para su mejora $\mathrm{y}$ perfeccionamiento.

De nuevo, en la conferencia, se planteó en el artículo 5. "Promover el saber mediante la investigación en los ámbitos de la ciencia, el arte y las humanidades y la difusión de sus resultados.

El progreso del conocimiento mediante la investigación es una función de todos los sistemas de educación superior. Debería fomentarse y reforzarse la innovación, la interdisciplinariedad y la transversalidad en los programas fundando las orientaciones a largo plazo en los objetivos y 
necesidades sociales y culturales (...) Se debería incrementar la investigación en todas las disciplinas, comprendidas las ciencias sociales y humanas, la educación (...) (UNESCO 1998:5-9).

Una de las vías para comprometer al profesional pedagógico con los problemas y su solución se da buscando la transformación de la realidad educativa hacia la calidad y cada vez más ajustada a las necesidades sociales y educativas. Un profesional comprometido con su realidad profesional asume la necesidad investigativa como generadora de cambio y la refleja en sus acciones, busca a cada paso la oportunidad aumentar el desempeño de sus tareas y funciones, lo que lo lleva a la innovación en su práctica docente. Una escuela que pretenda aspirar al reconocimiento mundial como es la de Angola necesita considerar, como una alternativa viable, desarrollar al individuo (tanto profesorado como alumnado), comprometido con los procesos educativos, pudiéndose lograr a través de la solución de problemas educativos mediante la investigación.

La UNESCO en su Declaración Mundial sobre Educación, afirma que:

Es necesario educar mediante experiencias de aprendizaje cooperativo y variedad de actividades pedagógicas centradas en el alumno. La habilidad de pensamiento crítico y las actitudes cooperativas, que estas apropiaciones desarrollan en los estudiantes y contribuyen a preparar a éstos para desempeñar mejor sus futuras funciones laborales, recuperar la práctica como espacio privilegiado de la formación y de reflexión. La práctica pedagógica es el proceso más importante, permanente y efectivo como lo advierten los propios docentes (2000:371).

Se destaca en esa propuesta la estrecha y necesaria relación teoríapráctica desde posiciones reflexivas y críticas en un proceso pedagógico democrático y cooperativo. Las anteriores afirmaciones de la UNESCO dan testimonio de que este órgano mundial ha sido persistente en el desarrollo de procesos de formación de profesores con orientación al desarrollo del pensamiento científico que sostiene y potencia la relación permanente entre la teoría y la práctica, lo que debe tenerse en cuenta en las IES y en la formación de profesores en particular.

Autores como Elliot, (1994); Stenhouse (1998); Munévar, 1998); Chirino, (2000); Muñoz, \& Quintero (2001); Pereira \& Zeichner (2002); Díaz et al. (2008), destacan la importancia de la formación en la investigación y la necesidad de añadirla a la docencia de forma permanente para asegurar el desarrollo profesional del docente y garantizar la formación integral de los estudiantes. En la senda de la formación investigativa de los profesores, fundamentada en la crítica reflexiva y el análisis científico como base en la orientación de los procesos pedagógicos, se evidencian otros 
autores como Sacristán, (1998); Imbermón (1997); Freire (1996); Pozo (1999) y Ramos (2010).

En Angola la preocupación de la formación en el ámbito de la investigación es antigua, está presente en las políticas del gobierno desde la creación de la Universidad de Angola. En este sentido, El Ministerio de la Educación (1978:24) en planteó: (...) "la Universidad de Angola será un medio privilegiado para la investigación científica, de la rama escogida, para que el conocimiento teórico adquirido sea siempre explicado por la investigación de la realidad nacional”. Sin embargo, en la práctica pedagógica no tuvo en sus momentos el reflejo esperado, aunque a más largo plazo se ha ido fortaleciendo la idea y se ha incentivado la búsqueda de alternativas y vías para su instrumentación.

En las Líneas Maestras de la Enseñanza Superior para la mejoría de la gestión del subsistema de la enseñanza superior, Nascimento (2005), destaca las siguientes:

- $\quad$ El contexto de paz en que se impone la promoción de la calidad de la vida de las poblaciones y de la normalidad del funcionamiento de las instituciones del estado, el sistema de educación y particularmente de enseñanza superior tiene un papel específico a desempeñar;

- $\quad$ Hay que apostar en la promoción de la calidad de un sistema de enseñanza superior que asegure una educación y una formación de ciudadanos que, por sus valores y sus competencias, tengan un impacto relevante tanto en su desarrollo personal como en el desarrollo de su comunidad y país, que contribuyan cada vez más para la afirmación nacional en la región de África Austral;

- Importa que esos aprendizajes se caractericen por su pertinencia, eficacia, que estén fundamentados en principios científicos, así como en valores universales, cívicos y morales compatibles con la cultura nacional angolana y con la orientación del desarrollo nacional;

- $\quad$ Lograr que la formación a ser promovida en el país sea el resultado de una búsqueda permanente del equilibrio entre los intereses, las necesidades y las posibilidades de los individuos, de las poblaciones y del estado;

- $\quad$ Es importante privilegiar una gestión que a todos los niveles coloque en el centro de su acción la noción de "escuela" que promueve la funcionalidad, la cualidad aceptable, posible y que, en consecuencia, ofrezca dignidad, orgullo a los angolanos y al país donde quiera que se preste un servicio del enseñanza superior.

- $\quad$ El plan de acción para la implementación de las líneas maestras para la mejora del subsistema de enseñanza superior, comprende un conjunto de acciones consideradas imprescindibles para corresponder las expectativas, de 
las cuales se destaca como una de las imperativas la promoción de la investigación científica, sobre todo en la aplicación de dicha investigación (Nascimento, 2006).

La importancia que se da a la investigación en la docencia inscrita en la enseñanza superior en Angola constituyó el eje central en II Encuentro de Rectores Angola-Cuba, realizado en Angola entre los días 10 y 11 de julio de 2013 con el lema "El Papel del docente universitario en la formación de cuadros de nivel Superior”. El Ministro de la Enseñanza Superior reforzó la visión de la urgencia de abordar la formación de los profesores para que, desde su labor profesional, tengan la capacidad de cumplir con los procesos sustantivos de docencia, investigación y extensión. Apeló a la comprensión de la responsabilidad de la enseñanza superior para que la visión del país se transforme en realidad. De esas ideas orientadoras, la gran expectativa recae sobre las instituciones de enseñanza superior, así como la responsabilidad social que tienen que asumir en el contexto nacional. Son las instituciones de la enseñanza superior las que tienen la responsabilidad de formar cuadros a nivel superior con el perfil que deben tener las mujeres y hombres en cuanto a ciudadanos, profesionales y peritos, funcionarios, profesores e investigadores. Toda esa política se concreta en la clase y en el resto de las actividades universitarias en las que el profesor debe orientar el proceso, para responder a las exigencias, en tanto el sustento básico debe ser la investigación permanente.

Así, las instituciones de enseñanza superior tienen la misión de Formar, Investigar, y Prestar Servicios a la Comunidad. Desde esa perspectiva las escuelas superiores pedagógicas siendo instituciones de enseñanza superior, tienen en general la misión estatuida por el órgano de tutela que es el Ministerio del Enseñanza Superior y para su cumplimento deben:

- $\quad$ Reunir y producir los saberes avanzados y más variados en el dominio de la ciencia, la técnica y la tecnología y dar solución a los problemas y desafíos que se colocan en los planes;

- Ofrecer al país los cuadros (profesores) necesarios capaces de responder a los desafíos del sistema de educación en general y en el área de formación en particular.

- $\quad$ El pleno cumplimiento de esta misión depende de la integración armoniosa de un conjunto de dispositivos educativos, entre ellos se destacan los cuatro componentes organizacionales del plan de estudio que deben interrelacionarse en el proceso de enseñanza y aprendizaje: académico, laboral, investigativo y extensionista, considerando que el investigativo debe mediar la relación entre ellos.

También se abordó el papel de la investigación científica en el proceso docente educativo destacándose que uno de los objetivos de la 
enseñanza superior es formar profesionales con competencias para proseguir sus acciones de postgrado, promover el desarrollo profesional continuo y aprender a lo largo de la vida. Los pronunciamientos expuestos anteriormente muestran la preocupación del órgano de tutela dela enseñanza superior en Angola, por potenciar la investigación con relevancia en la figura del profesor que es considerado pilar fundamental para la transformación deseada.

La investigación desarrolla en los profesores y en los estudiantes conocimientos, habilidades, actitudes y valores que impulsan su actualización permanente, la reflexión crítica, la adopción de métodos para la comprensión, transformación de la realidad y el aporte de soluciones de los problemas sobre la base de las evidencias de la propia ciencia y para la mejoría del proceso de enseñanza aprendizaje. Por eso, en las escuela superior pedagógica de Angola se está buscando generalizar y concientizar el abordaje del papel de la investigación científica en el proceso de enseñanza aprendizaje en el sentido de que no se debe limitar solamente a la enseñanza de las disciplinas que concentran los aspectos referentes a su metodología y la producción de trabajos o memoriales científicos y académicos insertados en ese limitado contenido, sino que se amplíe y contextualice a los problemas de la formación. En el contexto de formación y desarrollo de habilidades investigativas y de su uso como elemento integrador y facilitador del proceso de enseñanza aprendizaje es importante cuestionarse sobre los aportes que cada disciplina del currículo de graduación puede dar al perfil general del egresado en ese dominio (Zeichner \& Pereira, 2011).

Una de las situaciones que preocupa al órgano de tutela de la enseñanza superior, el Ministerio de la Enseñanza Superior, es el claustro de profesores de las IES, pues que en su mayoría no trabajan a tiempo completo e imparten clases a varias IES. En la Visión de Ministro de la Enseñanza Superior, ante esta situación, se considera pertinente el imperativo de trabajar en las siguientes prioridades: consolidar la visión estratégica de desarrollo de la enseñanza superior; reforzar la capacidad institucional, contando con los actores que participan e impulsan el desarrollo; directivos, profesores y estudiantes, con la calidad de procesos sustantivos desde una postura adecuada, con actitudes, comportamientos y valores; un ambiente académico exigente, así como el fomento de una cultura individual y colectiva, profesional e institucional, con sustento en la búsqueda permanente de la excelencia académica y de pertinencia social; exaltar ejemplos que sirvan de referencia y desaprobar en cualquier circunstancias los contra-ejemplos; instaurar la práctica de la investigación de los procesos en la evaluación de cada uno de los dispositivos y de cada IES, fundada en informaciones fiables (Nascimento, 2013). 
Además de lo expuesto anteriormente, las escuelas superiores pedagógicas de Angola tienen su situación muy complicada pues son instituciones nuevas cuyo claustro de profesores en tiempo integral está constituido mayoritariamente por docentes extranjeros de nacionalidad cubana, a lo cual se suma que los nacionales integrales son mayoritariamente licenciados con muy poco tiempo de experiencia en la docencia de la enseñanza superior.

En esos casos, una de las salidas inmediatas es la formación continua de los profesores universitarios. En esta vertiente, el profesor Peterson, P (2013) en la ponencia que hizo en el II Encuentro entre rectores de Angola y Cuba, destacó la necesidad de la formación continua de los profesores en al cual destacó las siguientes características esenciales: es dirigida a una población de profesores ya formados; tiene un contenido programático diferenciado, tiene por finalidad actualizar y perfeccionar profesionalmente al profesor, aplicar la investigación y divulgar la innovación educacional de modo permanente, y sobretodo evaluada por sus resultados. De esta manera se puede comprender que la formación continua es inseparable de la formación inicial. La formación continua de los profesores juega un papel crucial en la valorización de la profesión docente, en el desarrollo organizacional de las escuelas y en el mejoramiento de los aprendizajes de los estudiantes.

La otra situación, no menos importante es el hecho de que en las escuelas de formación de profesores no todos los que ingresan están interesados en la formación como profesores, unos ingresan porque es la única oportunidad que encuentran para hacer la formación superior. Otros porque para ingresar en el trabajo en la función pública en esa rama hay más facilidad; además, para cambiar de categoría y cobrar mayor salario se empeña en conseguir un diploma en la área de profesorado. En ese caso, muchos que buscan la escuela superior pedagógica, en particular la de Bié, son impulsados por poca motivación intrínseca para la profesionalización, o sea para profesar la profesión. Eso ha sido uno de los mayores problemas en el proceso de la formación de profesores, pues conducir el proceso ya es complejo, lo que se incrementa con una persona cuya motivación es extrínseca, por lo que el problema se agudiza. En esos casos, para los que son profesores de la Escuela Superior Pedagógica de Bié, el esfuerzo tiene que estar dirigido en función del desarrollo de intereses profesionales pedagógicos que favorezcan el cambio hacia una motivación intrínseca del proceso de formación y para ellos es necesario la investigación científica que les permita caracterizar sus estudiantes, identificar problemas pedagógicos, buscar alternativas que incentiven motivos profesionales, a partir de ejemplos positivos de profesional que investiga y desempeña sus funciones apoyándose en los recursos de la ciencia. 
En este caso es esencial la búsqueda y exploración de mecanismos para comprometer a las personas con el objeto de la profesión, en la formación de profesores. La vía para lograrlo pasa necesariamente por la investigación en el proceso pedagógico como ya se ha fundamentado.

Se considera que el aprendizaje y la adquisición de competencias en investigación educativa puede resumirse en: conocimiento científico pedagógico, que permite el análisis sistemático de la práctica educativa desde posiciones científicas y éticas; habilidades científico-investigativas que posibilitan operar con la ciencia en el contexto educativo; valores éticos profesionales que favorecen el compromiso consigo mismo y con la sociedad, estimulando el mejoramiento de la práctica educativa, la profesionalidad y la calidad humana de los participantes, se puede lograr con éxito la incorporación consciente, activa y comprometida de los que ingresan sintiéndose ajenos de la profesión, a partir de la cultura de valores éticos profesionales basados en la formación investigativa del educador que en su esencia, es un profesor investigador (de Jesús Paulo \& Chirino, 2011).

Con respecto a la ética profesional, en palabras de Monteiro (2004:175),

La finalidad de la formación de un profesor debería desarrollar sus conocimientos generales y su cultura personal; su capacidad para enseñar y para educar; su comprensión de los principios que presiden el establecimiento de buenas relaciones humanas en el interior y para allá de las fronteras nacionales; su conciencia del deber contribuir, al mismo tiempo, por la enseñanza y por el ejemplo, para el progreso social, cultural y económico.

En esa cita de Monteiro se puede ver cómo caracteriza la dimensión del valor ético de la profesionalidad del profesor desde una perspectiva transfronteriza que, sin el prudente condicionamiento en su formación, en la práctica es difícil lograrlo.

La formación investigativa del futuro profesional de la educación exige el desarrollo de valores éticos profesionales dentro de los cuales, revisten especial importancia el valor de la ciencia, el valor de la profesión y la honestidad científica, ya que debe partirse de que el futuro profesional busque en la ciencia las explicaciones a los hechos y fenómenos de la realidad educativa, que valore altamente la profesión pedagógica, se motive por el perfeccionamiento de la educación y tenga la honestidad para reconocer aciertos y errores, así como el carácter inacabado de la obra humana (Chirino, Paulo \& Crespo, 2012:21).

De ahí la importancia de lograr en los estudiantes intereses profesionales que condicionan su motivación y compromiso con la profesión.

Para eso es necesario que en el ámbito de la formación de los profesores en la Escuelas Superiores Pedagógicas de Angola los profesores 
se apropien del método científico para que puedan en el mismo ámbito formar los estudiantes. El método tiene un aspecto externo o fenomenológico y un aspecto interno o esencial que está íntimamente relacionado con los procesos mentales que realiza el estudiante. Por eso, en la Educación Superior los métodos deben ser productivos por norma ineludible, mucho más si de formación investigativa se trata. Estos métodos deben incentivar a los estudiantes a indagar, cuestionar la teoría y la práctica, buscar evidencias, elaborar hipótesis, aprobar, experimentar con sus posiciones científicas y desarrollar los procesos mentales que favorecen la solución de problemas (Chirino, 2002).

Aproximar la lógica del aprendizaje a la lógica de la investigación, implica un enfoque profesional del proceso de enseñanza - aprendizaje, lo cual presupone la necesidad de que este proceso parta de la delimitación de los problemas profesionales pedagógicos los que deben ser abordados en los diferentes años académicos graduando su complejidad y considerando el desarrollo alcanzado por los estudiantes.

Se está viendo el método en dos vertientes: método de enseñanzaaprendizaje y método científico. Cabe preguntarse si son conciliables, para eso hay que definir también el método científico para ver los nexos entre ambos, "el método científico se puede definir como forma de asimilación teórica y práctica de la realidad que parte de las regularidades del objeto de estudio o como el sistema de principios reguladores de la actividad transformadora, práctica, cognoscitiva y teórica” (Martínez Llantada, 2002: $3)$.

El objetivo del proceso de enseñanza-aprendizaje circunscrito a la investigación educativa, se encamina a la preparación del futuro profesor para el ejercicio de la función investigativa, prepararlo científicamente para las transformaciones educativas que demanda la escuela. El contenido del proceso de enseñanza-aprendizaje puede resumirse en conocimiento científico pedagógico, que permite el análisis sistemático de la práctica educativa desde posiciones científicas; habilidades científico-investigativas que posibilitan operar con la ciencia en el contexto educativo; valores éticos profesionales que favorecen el compromiso consigo mismo y con la sociedad, estimulando el mejoramiento de la práctica educativa, la profesionalidad y la calidad humana de los participantes desde el vínculo teoría-práctica que favorece la experiencia de la actividad creadora.

\section{Conclusion}

Luego del análisis de las tendencias actuales sobre la formación investigativa de los profesionales de la educación se evidencia:

1. En el contexto de Angola, en función de la problemática que se vive en las escuelas superiores pedagógicas, la formación investigativa 
constituye la salida para el comprometimiento profesional de futuros profesores, con dignificantes modos de actuación en la perspectiva de la transformación de la realidad educativa.

2. La formación investigativa del profesional de la educación proporciona los recursos teóricos y metodológicos para el cumplimiento de sus restantes funciones profesionales: función docente metodológica y función de orientación educacional.

3. La formación investigativa se torna una necesidad imperiosa para los maestros angolanos ya que contribuye a un cambio en su postura profesional, aumentando mayor responsabilidad y compromiso profesional en la labor que desempeñan.

\section{References:}

1. Castellanos, B. (1998). Investigación y cambio educativo en América Latina. Revista Varona,(26-27), 18-24.

2. Chirino, M. V. (2002). Perfeccionamiento de la formación inicial investigativa de los profesionales de la educación. [inédito], tesis de grado, Instituto Superior Pedagógico «Enrique José Varona», La Habana.

3. De Jesús Paulo, A. M., \& Chirino Ramo, M. V. (2012). El desarrollo de habilidades investigativas en las Universidades de Ciencias Pedagógicas de Cuba y Bié (Angola). In Revista Congreso Universidad (Vol. 1, No. 2, p. 15).

4. Chirino, M.; Paulo, A. \& Crespo, M. (2012). Propuesta Metodológica Para el Desarrollo de Habilidades Investigativas en la Escuela Superior Pedagógica de Bié, Angola. Comunicación en la Conferencia Científica UNICA 2012.

5. Danilov, M. A., \& Skatkin, M. N. (1978). Didáctica de la escuela media. Pueblo y Educación. Ciudad de La Habana.

6. Elliott, J. (1990). La investigación-acción en educación. Ediciones Morata.

7. Freire, P. (1996). Pedagogia da autonomia: saberes necessários à prática docente. São Paulo: Paz e Terra.

8. Díaz, T., Alfonso, P., Ruiz, J. M., González, M., González, J. R., \& González, M. (2008). La gestión universitaria y el rol del profesor. $L a$ Habana: Editorial Félix Varela.

9. Imbernón, F. (1997). La formación del profesorado: ciertas confusiones y algunas evidencias. Aula de innovación educativa, (62), 40-42.

10. Martínez Llantada, M. (2002). El método científico. In Conferencia magistral. Centro de Estudios Educacionales del Instituto Superior 
Pedagógico Enrique José Varona. Ciudad de La Habana. Versión Electrónica.

11. Monteiro, A. D. (2004). Educação e deontologia. Lisboa: Escolar Editora.

12. Munévar, R. A. (2001). Construcción de un currículo integrado por la educación superior (Doctoral dissertation, Tesis doctoral en ciencias pedagógicas. Cepes. Universidad de La Habana, Cuba y Universidad de Caldas-Colombia).

13. Muñoz, J., Quintero, J., \& Munévar, R. (2001). Como desarrollar competencias investigativas en educación. Consultado en Marzo, 28.

14. Pereira, J. E. D. \& Zeichner, K. M. (2002). A pesquisa na formação e no trabalho docente. Belo Horizonte: Autêntica, 46.

15. Pozo, J. I. (1999). Aprendices y maestros: la nueva cultura del aprendizaje. Alianza Editorial.

16. Ramos, K. M. (2010). Reconfigurar a profissionalidade docente universitária: um olhar sobre ações de atualizações de atualização pedagógico-didática. U. Porto.

17. Ramos, S. \& Naranjo, E. (2014). Metodologia da Investigação Científica. Escolar Editora. Lobito-Angola.

18. Stenhouse, L. (1998). La investigación como base de la enseñanza. Ediciones Morata.

19. UNESCO. (1998). Debate Temático: Educación Superior Para Una Nueva Sociedad. La Visión de los Estudiantes. Conferencia Mundial Sobre la Educación Superior, La Educación Superior en el Siglo XXI: Visión y Acción”. Paris, 5-9 de octubre.

20. UNESCO. (1998). Declaración Mundial Sobre la Educación Superior en el Siglo XXI: Visión y Acción. Conferencia Mundial Sobre la Educación Superior, Paris, 5-9 de octubre.

21. UNESCO. (2000). Declaración Mundial Sobre Educación al Siglo XXI. 